\title{
Próteses auditivas: estudo comparativo das dificuldades auditivas e do benefício da amplificação em pacientes usuários de amplificação não linear e linear
}

\author{
Érika Cristina Bucuvic* \\ Maria Cecília Martinelli Iório**
}

\begin{abstract}
Resumo
O objetivo deste trabalho foi quantificar e comparar as dificuldades auditivas e o benefício da amplificação em novos usuários de aparelho auditivo segundo as variáveis tipo de amplificação linear e não linear e tempo de uso, por meio do questionário de auto-avaliação APHAB. Foram avaliados 42 pacientes adultos com perda auditiva de grau leve a severo, distribuídos de acordo com o tipo de amplificação utilizada, sendo 13 pacientes usuários de amplificação não linear e 29 pacientes usuários de amplificação linear. O questionário de auto-avaliação APHAB, desenvolvido por Cox e Alexander em 1995, foi aplicado aos pacientes dos diferentes grupos antes da adaptação e após dois e seis meses. Para a análise estatística, foi utilizado o teste ANOVA com nível de significância de 5\%. Os pacientes usuários de circuitos de amplificação linear apresentaram melhor desempenho nas subescalas FC (facilidade de comunicação), RV (reverberação) e BA (barulho ambiente), não sendo esta diferença estatisticamente significativa. O benefício obtido após dois e seis meses de uso da amplificação foi satisfatório. O desconforto para sons intensos, subescala (SI), foi maior para os pacientes usuários de amplificação linear. Não foram constatadas diferenças significativas no desempenho de pacientes usuários de circuitos de amplificação linear e não linear, porém houve uma tendência de os pacientes usuários de amplificação linear apresentarem menor dificuldade em diferentes ambientes acústicos. O benefício com o uso do aparelho de amplificação é satisfatório independentemente do tipo de amplificação e do tempo de uso.
\end{abstract}

Palavras-chave: Auxiliares da audição. Perda auditiva. Auto-avaliação. APHAB.

\section{INTRODUÇÃO}

O benefício obtido com a amplificação é a diferença entre o desempenho do paciente com e sem a prótese auditiva. Este benefício obtido com próteses auditivas tem sido tradicionalmente medido por meio de testes objetivos, como ganho funcional (diferença de limiares auditivos sem e com o uso da prótese auditiva), inteli- gibilidade de fala e crescimento da sensação de intensidade. Recentemente, tem se observado que, se, por um lado, estas medidas são úteis para verificar a adequação do processamento do sinal acústico, por outro, o seu papel para validar a amplificação é questionável. Assim sendo, pode-se ter uma prótese auditiva fornecendo uma boa audibilidade, mas as dificuldades auditivas e psicossociais podem ser consideráveis,

\footnotetext{
* Fonoaudióloga. Mestre em Ciências dos Distúrbios da Comunicação Humana. Escola Paulista de Medicina.

UNIFESP. Fga do Setor de Audiologia da FUNCRAF - subsede Santo André.

Escola Paulista de Medicina

Universidade Federal de São Paulo

Rua Andaraí, 500 ap 84

09.050-000 Santo André São Paulo Brasil

E-mail:bucuvic@ig.com.br

** Fonoaudióloga. Professora Doutora da Disciplina dos Distúrbios da Audição. Escola Paulista de Medicina. UNIFESP.
} 
enquanto uma outra pode fornecer apenas um pequeno percentual de fala audível mas com considerável redução das dificuldades auditivas e desvantagens psicossociais. Em vista dessa pobre relação entre as medidas objetivas e aquelas baseadas na percepção do paciente, as medidas subjetivas vêm ganhando aceitação, sendo um componente importante no processo de adaptação da prótese auditiva (WEINSTEIN, 1997).

Até meados da década de 90, os sistemas de amplificação processavam a maioria dos sinais de entrada de forma linear, sendo, a única distinção entre os diferentes tipos de sistemas de amplificação, o modo como era limitada a saída máxima (corte de picos - PC e limitação por compressão). A partir dessa época, em função do conhecimento da não linearidade da cóclea, foram introduzidos os sistemas não lineares que processam o sinal de fraca e média intensidade (fala) com ganho variável. Atualmente, a classificação clínica das próteses auditivas vem ocorrendo segundo o modo como a fala é processada; a fala possui uma intensidade média de 60 a 65 dB NPS, com picos de máxima ou mínima intensidade variando cerca de 30 $\mathrm{dB}$. As próteses auditivas que processam os sons médios de forma linear (com ganho constante) vêm sendo denominadas "próteses lineares"; já aquelas que processam a fala de forma não linear (com ganho variável) vêm sendo denominadas "não lineares" ou próteses auditivas com compressão dinâmica - Wide Dynamic Range Compression (WDRC) - (MENEGOTTO; IÓRIO, 2003*). Novos métodos de avaliação foram necessários para a prescrição e avaliação da nova tecnologia, considerando principalmente a nova geração de aparelhos não lineares com compressão dinâmica (WDRC).

Com esta finalidade, foi introduzido formalmente, em 1994, um fórum chamado Independent Hearing Fitting Fórum (IHAFF) - Fórum Independente de Seleção/Adaptação de Aparelhos Auditivos —, que teve como principal objetivo discutir as etapas do processo de seleção e indicação de aparelhos auditivos, especialmente não lineares. A meta estabelecida pelo protocolo foi a de normalizar o crescimento da sensação de intensidade dos pacientes. $\mathrm{Na}$ época, as fórmulas prescritivas eram apropriadas para a adaptação de aparelhos lineares e não eram apropriadas para a seleção de um aparelho não linear. $O$ fórum estabeleceu etapas importantes deste processo, recomendando a aplicação do questionário de auto-avaliação $\mathrm{APHAB}$ Abbreviate Profile of Hearing Aid Benefit (COX; ALEXANDER, 1995) antes do uso da prótese auditiva e na validação dos resultados da adaptação (VALENTE; VLIET, 1997).

$\mathrm{Na}$ literatura, grande número de pesquisas têm sido realizadas utilizando questionários de auto-avaliação, sendo um dos objetivos o de avaliar e comparar diferentes tecnologias (BORTHOLUZZI, 1999; BENTLER; DUVE, 2000; NEWMAN, 2000; YUED, 2000).

A análise das dificuldades auditivas frente às diferentes situaçôes de comunicação, com diferentes circuitos de amplificação, é imprescindível para modificar indicaçôes, avaliar o tratamento e propor mudanças.

O presente estudo tem como objetivo quantificar e comparar as dificuldades auditivas e o benefício da amplificação em novos usuários de aparelho auditivo, segundo as variáveis tempo de uso e tipo de amplificação linear e não linear, por meio do questionário de auto-avaliação APHAB.

\section{MATERIAIS E MÉTODOS}

Os indivíduos portadores de deficiência auditiva que compuseram a casuística do presente estudo foram atendidos no programa de reabilitação da Fundação para o Estudo e Tratamento das Deformidades Craniofaciais (FUNCRAF), subsede Santo André, nos anos de 2001 e 2002. Os pacientes foram convoca-

\footnotetext{
* MENEGOTTO, I. H; IÓRIO, M. C. M. Processamento dos sinais sonoros nas próteses auditivas: amplificação linear e não linear (ou compressão). In: ALMEIDA, K.; IÓRIO, M. C. M. Próteses auditivas: fundamentos teóricos e aplicaçōes clínicas. São Paulo: Lovise, 2003. No prelo.
} 
dos para iniciar o tratamento com base em uma lista de espera previamente estabelecida pela instituição.

\section{Casuística}

A casuística foi constituída por 42 indivíduos, $26(61,9 \%)$ do sexo feminino e 16 $(38,1 \%)$ do sexo masculino, de faixa etária de 20 a 76 anos (média de 53 anos). Quanto ao nível de escolaridade, $52,4 \%$ dos pacientes apresentavam ensino fundamental incompleto; $16,6 \%$, nível de ensino fundamental; $7,2 \%$, ensino médio incompleto; $21,4 \%$, ensino médio; e 2,4\%, ensino superior. A classificação quanto ao nível socioeconômico variou de baixo inferior a médio inferior. Dos indivíduos selecionados, 29 (69\%) eram portadores de deficiência auditiva neurossensorial e 13 (31\%) de deficiência auditiva mista bilateral de grau leve a severo bilateral.

A adaptação foi binaural em todos os casos. As marcas variaram de um paciente para outro. O uso do circuito de amplificação linear compreendeu $69 \%$ da amostra e o não linear $31 \%$. O tempo de uso diário foi, em média, de 8 horas e 18 minutos (DP 2,08).

\section{Procedimentos}

Após avaliação otorrinolaringológica, audiológica e processo de seleção / indicação da prótese auditiva, os pacientes selecionados foram submetidos à avaliação do benefício da amplificação por meio da aplicação do questionário de auto-avaliação APHAB desenvolvido por Cox e Alexander em 1995 (ANEXO 1). Este foi aplicado antes da adaptação da prótese auditiva e após dois e seis meses de uso das mesmas.

O questionário APHAB quantifica dificuldades (incapacidades) auditivas, vivenciadas no dia-a-dia em diferentes situações de comunicação, incluindo aquelas em ambientes favoráveis e as vivenciadas na presença de ruído e salas reverberantes. Avalia, além disso, a tolerância a sons intensos do ambiente. É composto por 24 perguntas divididas em quatro subescalas de avaliação: 1) facilidade de comunicação (FC) comunicação em ambientes relativamente favoráveis; 2) reverberação (RV) - comunicação em salas e classes reverberantes; 3) ruído ambiental (BA) - comunicação em situaçôes com elevado nível de ruído de fundo; e 4) sons indesejáveis (SI) - desconforto aos sons intensos do ambiente. Para cada subescala, há seis perguntas distribuídas aleatoriamente. Cada questão descreve uma situação de comunicação específica, e são apresentadas duas colunas de resposta: (i) sem o uso do aparelho auditivo e (ii) com o uso do aparelho auditivo. Nas respostas, o paciente deve decidir com qual frequêencia a situação proposta ocorre e classificá-la dentre uma lista de sete alternativas. Cada alternativa é apresentada de forma descritiva, associada a uma porcentagem para auxiliar o paciente na sua interpretação: "sempre" (99\%), "quase sempre" (87\%), "geralmente" (75\%), "metade das vezes" (50\%), "às vezes" (25\%), "raramente" (12\%) e "nunca" (1\%). As respostas podem ser obtidas utilizando o questionário impresso ou diretamente no software do questionário. Para o presente estudo foi utilizado o material impresso.

Os questionários foram aplicados antes da adaptação do aparelho auditivo e após dois e seis meses de uso da amplificação. Foi introduzida uma terceira coluna de respostas para o preenchimento após seis meses.

Os pacientes foram divididos em dois grupos de acordo com o tipo de amplificação linear e não linear. Foram considerados sistemas lineares as próteses auditivas com corte de picos e com limitação por compressão, e não lineares aquelas com compressão dinâmica, independentemente do tipo de tecnologia (analógica ou digital).

O grupo não linear foi composto por 13 pacientes e o linear por 29. Vale ressaltar que os pacientes do grupo linear utilizavam aparelhos analógicos com limitação por compressão e/ou corte de picos e os pacientes do grupo não linear faziam uso de aparelhos digitais com compressão dinâmica (WDRC). Coincidentemente, todos os sistemas não lineares utilizados possuíam tecnologia digital. 
As respostas do questionário foram quantificadas por um programa disponibilizado pela empresa PHONAK em seu software de programação dos aparelhos auditivos.

A interpretação dos resultados foi baseada na análise das respostas relativas à condição sem prótese auditiva (antes da adaptação) e com prótese auditiva (após dois e seis meses de uso) nas subescalas: FC - facilidade de comunicação, RV - reverberação e BA - barulho ambiente. Segundo os critérios de análise dos autores do questionário, quando for considerada uma subescala individualmente, uma diferença de vinte e dois pontos entre a condição sem aparelho e com aparelho de amplificação sonora é considerada significativa; se o interesse for uma avaliação global das subescalas, uma diferença de até cinco pontos está demonstrando uma melhora na performance com o aparelho de amplificação sonora, e a chance de erro é uma em dez; uma diferença de dez pontos entre todas as subescalas (FC, RV e BA) representa um benefício real, e a chance de erro é de quatro em 100. A subescala SI ainda não está bem compreendida, e os autores sugerem a realização de um maior número de pesquisas antes de agrupá-la com as demais subescalas. Alguns pesquisadores têm tido sucesso utilizando os resultados desta subescala como base para ajustar os níveis de saída máxima (COX; ALEXANDER, 1995). Na subescala SI, Cox (1997) considerou significativa uma diferença maior do que ou igual a 31 pontos.

No presente estudo, foi realizada a comparação das dificuldades auditivas e a análise do benefício segundo os critérios propostos por Cox e Alexander (1995).

\section{Análise estatística}

$\mathrm{Na}$ comparação entre os grupos foi utilizada a técnica conhecida como Comparação de
Médias Univariada (ANOVA). O critério para determinar a significância estatística foi de $\mathrm{p}$ (nível descritivo) menor do que ou igual a 5\%. Os valores significativos são assinalados com asterisco.

\section{RESULTADOS}

Após a distribuição dos 42 pacientes pelos dois grupos (não linear $=13$ e linear $=29$ ), realizou-se o levantamento das dificuldades auditivas dos pacientes usuários de amplificação não linear e linear antes da adaptação da prótese auditiva. Os valores proporcionais médios dessas dificuldades por subescalas podem ser observados no Quadro 1.

\begin{tabular}{c|cccc}
\hline \multirow{2}{*}{$\begin{array}{c}\text { Tipo de } \\
\text { amplificação }\end{array}$} & \multicolumn{4}{|c}{ Dificuldades auditivas } \\
\cline { 2 - 5 } & FC & RV & BA & SI \\
\hline $\begin{array}{c}\text { Não linear } \\
(13)\end{array}$ \\
$\begin{array}{c}\text { Linear } \\
(29)\end{array}$ & 0,70 & 0,58 & 0,66 & 0,39 \\
\hline
\end{tabular}

QUADRO 1 - Valores proporcionais médios das dificuldades auditivas dos pacientes dos grupos usuários de amplificação não linear e linear

A seguir, calculou-se a proporção média das dificuldades auditivas nos diferentes grupos após dois e seis meses de adaptação, e realizou-se o estudo comparativo por meio do teste estatístico ANOVA. As Tabelas $1 \mathrm{e}$ 2 apresentam, respectivamente, os resultados deste estudo. As Figuras 1 e 2 ilustram os mesmos. 
TABELA 1

Valores proporcionais médios das dificuldades auditivas nas subescalas FC, RV, BA, SI do questionário $\mathrm{APHAB}$, nos grupos de usuários de amplificação não lienar $(\mathrm{n}=13)$ e linear $(n=29)$, após dois meses de adaptação e resultados do teste ANOVA

\begin{tabular}{lcccccccc}
\hline 2 meses & FC & \multicolumn{1}{c}{ RV } & \multicolumn{2}{c}{ BA } & \multicolumn{2}{c}{ SI } \\
& Não linear & Linear & Não linear & Linear & Não linear & Linear & Não linear & Linear \\
\hline Média & 0,28 & 0,26 & 0,37 & 0,25 & 0,42 & 0,34 & 0,36 & 0,48 \\
Desvio padrão & 0,18 & 0,21 & 0,27 & 0,16 & 0,21 & 0,16 & 0,2 & 0,27 \\
Tamanho & 13 & 29 & 13 & 29 & 13 & 29 & 13 & 29 \\
p-valor & \multicolumn{2}{c}{0,755} & \multicolumn{2}{c}{0,037} & & 0,182 & & 0,16 \\
\hline
\end{tabular}

FC $=$ Facilidade de comunicação

$\mathrm{RV}=$ Reverberação

$\mathrm{BA}=$ Barulho ambiente

SI = Sons indesejáveis

TABELA 2

Valores proporcionais médios das dificuldades auditivas nas subescalas FC, RV, BA, SI do questionário $\mathrm{APHAB}$, nos grupos de usuários de amplificação não linear $(n=13)$ e linear $(n=29)$, após seis meses de adaptação e resultados do teste ANOVA

\begin{tabular}{lcccccccc}
\hline 6 meses & FC & \multicolumn{2}{c}{ RV } & BA & \multicolumn{2}{c}{ SI } \\
& Não linear & Linear & Não linear & Linear & Não linear & Linear & Não linear & Linear \\
\hline Média & 0,21 & 0,18 & 0,27 & 0,21 & 0,31 & 0,25 & 0,31 & 0,35 \\
Desvio padrão & 0,18 & 0,16 & 0,14 & 0,16 & 0,14 & 0,14 & 0,22 & 0,23 \\
Tamanho & 13 & 29 & 13 & 29 & 13 & 29 & 13 & 29 \\
p-valor & \multicolumn{2}{c}{0,551} & \multicolumn{2}{c}{0,246} & & 0,202 & 0,579 \\
\hline
\end{tabular}

FC = Facilidade de comunicação

$\mathrm{RV}=$ Reverberação

$\mathrm{BA}=$ Barulho ambiente

$\mathrm{SI}=$ Sons indesejáveis

Os usuários de amplificação linear apresentaram dificuldades auditivas significativamente menores do que os usuários de amplificação não linear na subescala RV após dois meses de adaptação da prótese auditiva. Após seis meses de adaptação, não houve diferenças entre os grupos.

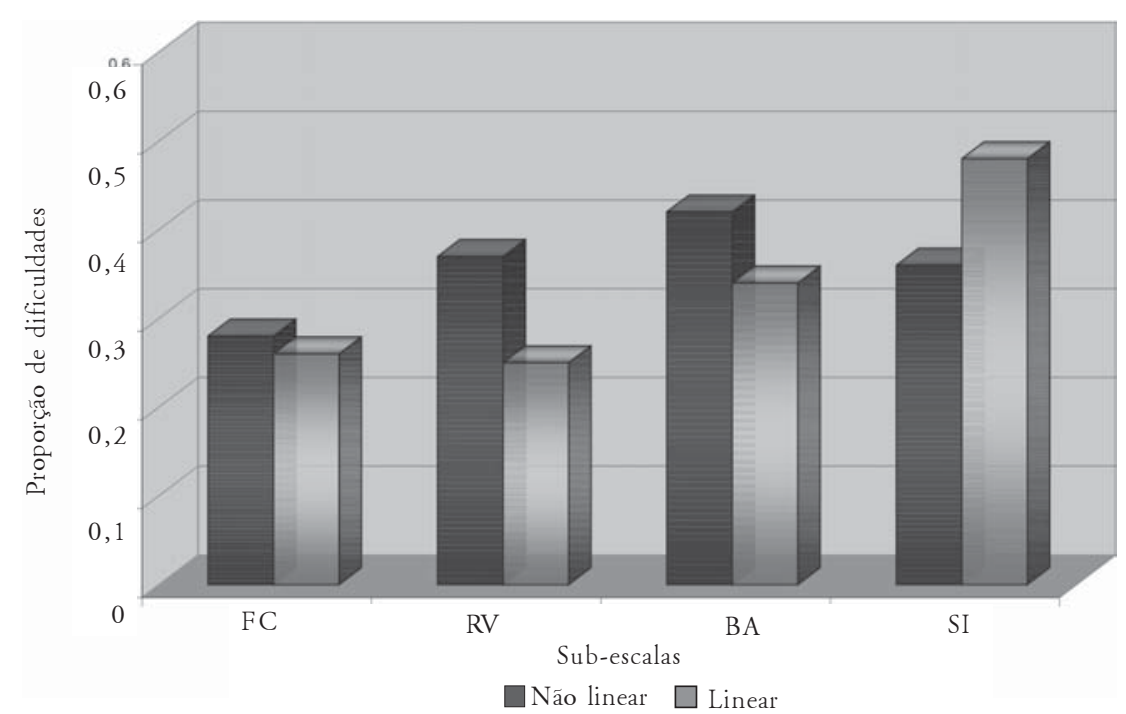

FIGURA 1 - Valores proporcionais médios das dificuldades auditivas dos usuários de amplificação não linear ( $\mathrm{n}=13$ ) e linear ( $\mathrm{n}=29)$, após dois meses de adaptação 


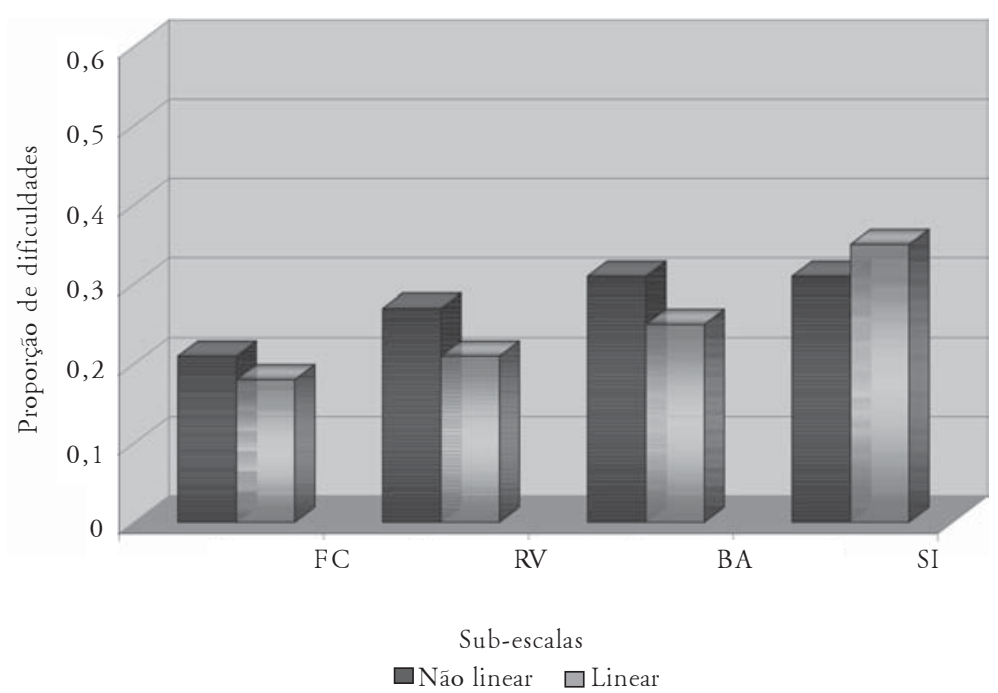

FIGURA 2 - Valores proporcionais médios das dificuldades auditivas dos usuários de amplificação não linear ( $\mathrm{n}=13)$ e linear ( $\mathrm{n}=29)$, após seis meses de adaptação

Realizou-se, em seguida, nos dois grupos, o estudo do benefício subjetivo obtido com o uso da amplificação não linear e linear após dois e seis meses de adaptação. Os valores percentuais mé- dios do benefício com o uso do aparelho após dois e seis meses podem ser observados nas Tabelas $3 \mathrm{e}$ 4. As Figuras 3 e 4 representam os gráficos dos valores percentuais médios do benefício.

\section{TABELA 3}

Benefício médio (\%) dos pacientes usuários de amplificação não linear $(n=13)$, após dois e seis meses de uso do aparelho auditivo

\begin{tabular}{lcccc}
\hline Período & \multicolumn{4}{c}{ Benefício - Grupo não linear } \\
\cline { 2 - 5 } & FC & RV & BA & SI \\
\hline 2 meses & $42 \%$ & $29 \%$ & $28 \%$ & $3 \%$ \\
6 Meses & $49 \%$ & $39 \%$ & $39 \%$ & $8 \%$ \\
\hline
\end{tabular}

FC = Facilidade de comunicação

$\mathrm{RV}=$ Reverberação

$\mathrm{BA}=$ Barulho ambiente

SI = Sons indesejáveis

TABELA 4

Benefício médio (\%) dos pacientes usuários de amplificação não linear $(n=29)$, após dois e seis meses de uso do aparelho auditivo

\begin{tabular}{crcrr}
\hline & \multicolumn{4}{c}{ Benefício - Grupo linear } \\
\cline { 2 - 5 } Período & FC & RV & BA & SI \\
\hline 2 meses & $42 \%$ & $46 \%$ & $34 \%$ & $-14 \%$ \\
6 meses & $50 \%$ & $49 \%$ & $45 \%$ & $-1 \%$ \\
\hline
\end{tabular}

$\mathrm{FC}=$ Facilidade de comunicação

$\mathrm{RV}=$ Reverberação

$\mathrm{BA}=$ Barulho ambiente

SI = Sons indesejáveis 


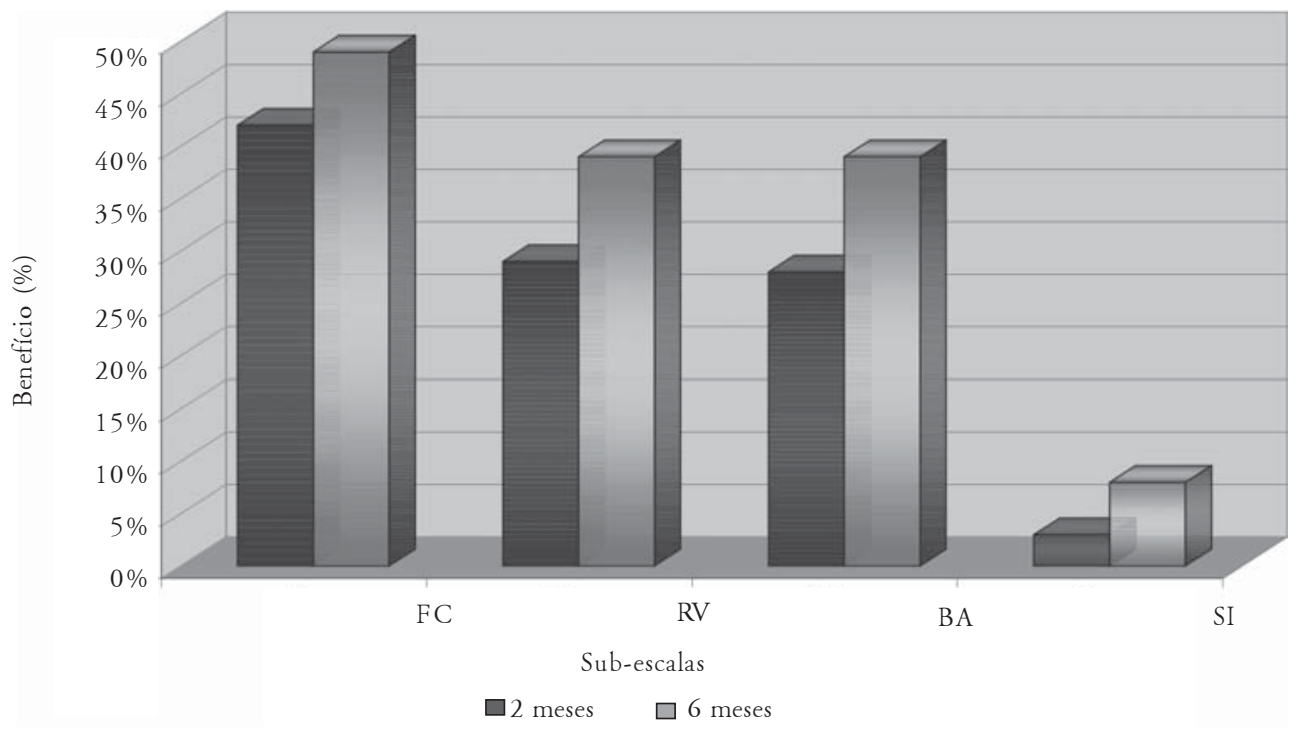

FIGURA 3 - Benefício médio (\%) após dois e seis meses de uso do aparelho de amplificação sonora no grupo usuário de amplificação não linear

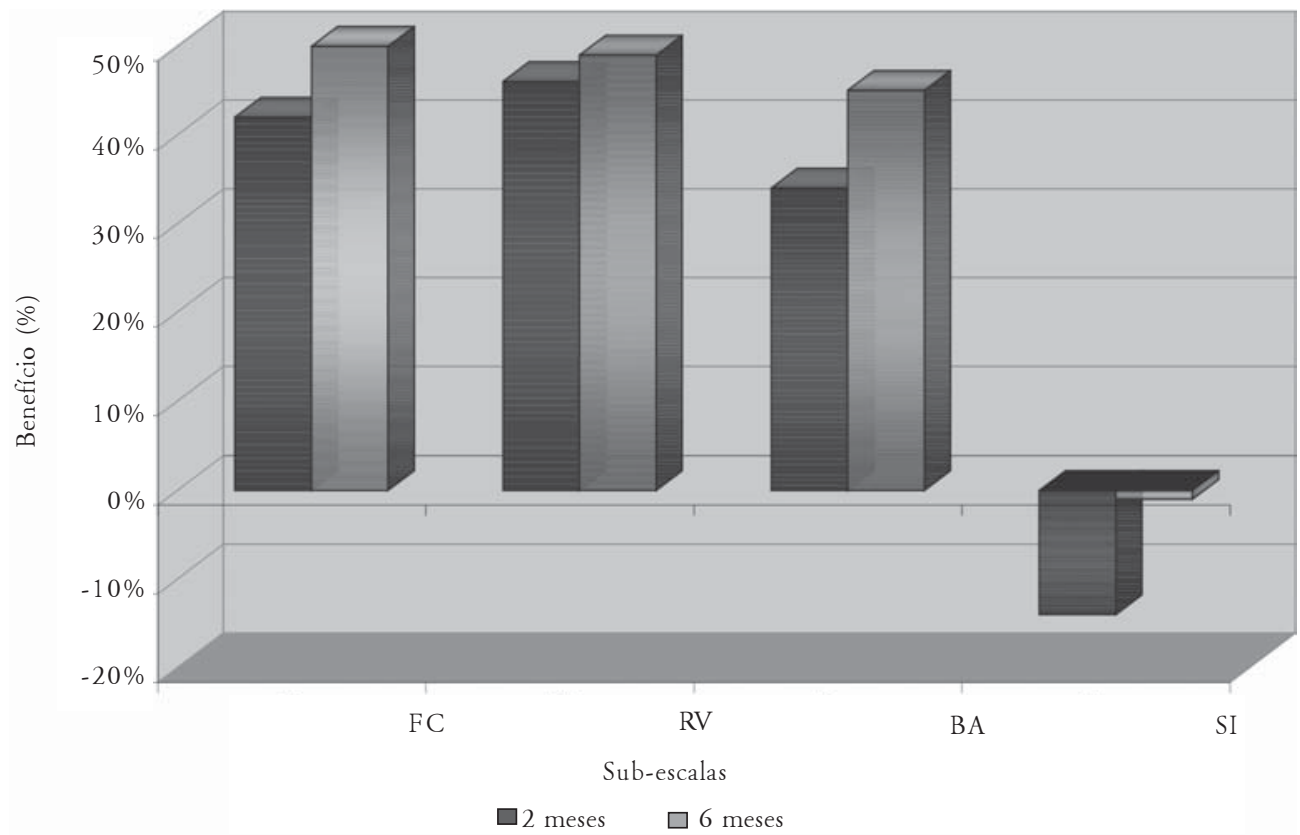

FIGURA 4 - Benefício médio (\%) após dois e seis meses de uso do aparelho de amplificação sonora no grupo usuário de amplificação linear 
Considerando os critérios de Cox e Alexander, observou-se que houve benefício satisfatório nas subescalas FC, RV e BA após dois e seis meses de adaptação tanto para o grupo usuário de amplificação linear como para o grupo não linear.

\section{DISCUSSÃO}

As dificuldades dos pacientes usuários de amplificação não linear $(n=13)$ e linear $(n=29)$ foram comparadas após dois e seis meses de adaptação.

A proporção média de dificuldades auditivas vivenciadas em ambientes favoráveis à comunicação, em ambientes e salas reverberantes e em ambientes com a presença de ruído de fundo - representadas, respectivamente, pelas subescalas FC (facilidade de comunicação), RV (reverberação) e BA (barulho ambiente) do questionário $\mathrm{APHAB}$ - , pelos pacientes usuários de amplificação não linear (NL), após dois meses de adaptação, variou de 0,28 a 0,42 . Isto significa que os mesmos apresentavam uma dificuldade auditiva para essas situaçôes de $28 \%$ a 42\%. Já os pacientes usuários de amplificação linear apresentaram dificuldades auditivas menores, tendo seus valores proporcionais médios variado de 0,25 a 0,34 (25\% a $34 \%)$, ou seja, os pacientes usuários de amplificação não linear apresentavam até $42 \%$ de dificuldades auditivas e os pacientes usuários de amplificação linear apresentavam até $34 \%$, considerando as diferentes situações avaliadas (TABELA 1). Após seis meses de adaptação, pode-se verificar diminuição das dificuldades auditivas para estas situaçôes tanto no grupo de pacientes usuários de amplificação não linear como no grupo de pacientes usuários de amplificação linear (TABELA 2).

$\mathrm{Na}$ comparação entre os grupos nessas subescalas, os usuários de amplificação linear apresentaram dificuldades auditivas significativamente menores do que os usuários de amplificação não linear apenas na subescala RV após dois meses de adaptação. Após seis meses, nenhuma diferença significativa foi observada entre os grupos.
Os achados de Walden et al. (2000) foram diferentes dos encontrados no presente estudo. Os autores verificaram melhor desempenho com a tecnologia não linear digital quando comparada à tecnologia analógica com limitação por compressão, porém, em circuitos analógicos com compressão dinâmica (WDRC) e digitais, não foram observadas diferenças de desempenho.

Nas situaçôes relacionadas com a intolerância para sons intensos ("sons indesejáveis"), subescala SI, as dificuldades auditivas do grupo usuário de amplificação linear após dois meses de adaptação foram maiores, com valores proporcionais médios de 0,48 ; já no grupo usuário de amplificação não linear, os valores proporcionais médios foram menores ( $\mathrm{SI}=0,36)$, ou seja, os pacientes usuários de amplificação linear apresentaram maior desconforto para sons intensos “indesejáveis” do que os usuários de amplificação não linear no início do uso da prótese auditiva. Estatisticamente, não houve diferenças significativas entre os tipos de amplificação nesta subescala (TABELA 1).

Após seis meses de uso da amplificação, nenhuma diferença significativa entre os grupos foi observada nas subescalas FC, RV, BA e SI do questionário APHAB. Vale ressaltar que houve uma redução das dificuldades auditivas em ambos os grupos nas quatro subescalas do questionário, porém o grupo usuário de amplificação linear manteve índices menores de dificuldades, quando comparado ao grupo usuário de amplificação não linear, para todas as subescalas. Na subescala SI, os valores proporcionais médios das dificuldades do grupo usuário de amplificação linear diminuíram de 0,48 para 0,35 , demonstrando que, ao longo do tempo de adaptação, sentem menor desconforto para sons intensos do ambiente, atingindo índices semelhantes aos dos pacientes usuários de amplificação não linear após seis meses de uso (TABELA 2).

Diferenças significativas entre as tecnologias também não foram encontradas em outros estudos. Nos estudos de Valente et al. (1997), Gordo (1998) e Bortholuzzi (1999), também não foram evidenciadas diferenças sig- 
nificativas entre os pacientes usuários de diferentes circuitos de amplificação. Valente et al. (1997) comparou, por meio de medidas subjetivas, dois tipos de aparelhos: linear com limitação por compressão, e não linear (WDRC) digitalmente programável; Gordo (1998) e Bortholuzzi (1999) compararam os circuitos analógico e digital por meio de medidas objetivas e subjetivas. Como resultados, não encontraram diferenças significativas com relação ao reconhecimento de fala no ruído e nas diferentes subescalas do questionário APHAB. Foram evidenciadas apenas diferenças significativas no ganho funcional. Bentler e Duve (2000), por outro lado, não observaram diferenças significativas entre diferentes tipos de tecnologia, por meio de testes objetivos e subjetivos.

$\mathrm{Na}$ literatura, a superioridade da tecnologia digital não é comprovada de maneira eficiente. Alguns autores, como Newman (2000) e Yued (2000), consideram falhas as pesquisas que têm como objetivo comparar as diferentes tecnologias (analógica e digital) e com grandes contrastes metodológicos.

\section{Benefício}

O benefício subjetivo adquirido após dois e seis meses de adaptação do aparelho auditivo foi satisfatório nos pacientes dos dois grupos estudados (não linear e linear). Considerando os critérios de Cox e Alexander (1995), observou-se uma diferença acima de 22 pontos percentuais nas subescalas FC, RV e BA. Com relação à subescala SI, o benefício médio foi insatisfatório tanto para o grupo usuário de amplificação não linear como para o grupo linear, porém os pacientes usuários de amplificação linear apresentaram benefício negativo após dois e seis meses de uso do aparelho auditivo, indicando maior desconforto auditivo para sons intensos do ambiente do que os pacientes usuários de amplificação não linear (TABELAS 3 e 4).

Considerando cada grupo isoladamente, foi possível observar, após seis meses de uso do aparelho de amplificação sonora, maior benefício nas quatro situaçôes de comunicação avaliadas pelo questionário: aquelas em ambientes favoráveis (FC), em ambientes e salas reverberantes (RV), em ambientes ruidosos (BA) e em ambientes com a presença de sons intensos "indesejáveis" (SI). Já com relação ao benefício adquirido com o uso da amplificação linear, observaram-se as mesmas características do grupo não linear nas subescalas $\mathrm{FC}, \mathrm{RV}$ e BA - benefício satisfatório e maior com maior tempo de adaptação (seis meses) —, porém, nas situações com a presença de sons intensos, representadas pela subescala SI, foram constatados valores negativos e redução desses valores após seis meses de adaptação, indicando uma diminuição da intolerância para sons intensos "indesejáveis" após este tempo (TABELA 4).

$O$ benefício adquirido em ambientes relativamente favoráveis à comunicação (subescala FC) foi semelhante tanto para o grupo de pacientes usuários de amplificação não linear como para o de pacientes usuários de amplificação linear, considerando os períodos de dois e seis meses. No início da adaptação (após dois meses), o benefício dos pacientes usuários de amplificação não linear foi menor em ambientes reverberantes (RV) e em ambientes ruidosos (BA) comparado ao benefício adquirido pelos pacientes usuários de amplificação linear no mesmo período de avaliação; após seis meses de adaptação, observou-se um maior benefício para estas situaçōes (RV e BA). Já os pacientes usuários de amplificação linear apresentaram grande benefício desde o início da adaptação (após dois meses).

\section{CONCLUSÕES}

Não há diferenças entre o desempenho de pacientes usuários de circuitos de amplificação não linear e linear, porém há uma tendência de os pacientes usuários de amplificação linear apresentarem menor dificuldade. Há redução progressiva das dificuldades auditivas após dois e seis meses de uso da amplificação, independentemente do tipo de circuito de amplificação. $O$ benefício com o uso do aparelho de amplificação é satisfatório. 


\title{
Hearing aids: comparative study of the hearing difficulties and the benefit of the amplification in hearing aids users with non-linear and linear amplification
}

\begin{abstract}
The purpose of this study was to quantify and to compare the hearing difficulties and the benefit of the amplification in new hearing aid users, through application of APHAB selfscale questionnaire, taking into consideration two variables: the linear or non-linear amplification type and the time of use. Forty-two patients with mild to severe hearing losses were evaluatedthirteen of them with non-linear amplification and twenty-nine with linear amplification. The APHAB self scale questionnaire, developed by Cox and Alexander in 1995, was filled in by the patients of the different groups before the hearing aid was fitted and, also, after two and six months of its use. For the statistical analysis, the ANOVA test was used with a significance level of 5\%. The linear hearing aids users showed better performance in EC (Ease of Communication), RV (Reverberation) and BN (Background Noise) subscales, although those results have not been statistically significant. The acquired benefit of the amplification after two and six months of the hearing aid use was satisfactory. The discomfort for loud sounds (AV subscale) was greater for the linear hearing aid users. Significant differences were not observed in the performance of the users of linear and non linear circuits, however there was some tendency for the linear hearing aid users to present less difficulty in different acoustic situations. The benefit obtained from the amplification was satisfactory, independently of the hearing aid type and the time of its use.
\end{abstract}

Keywords: Hearing aids. Hearing loss. Self-assessment. APHAB.

\section{REFERÊNCIAS}

BENTLER, R. A; DUVE, M. R. Comparison of hearing aids over the $20^{\text {th }}$ century. Ear Hear, v.21, p.625-639, 2000 .

BORTHOLUZZI, S. M. F. Estudo comparativo do desempenho das próteses auditivas analógicas e digitais em indivíduos adultos. 1999. Tese (Doutorado) - Universidade Federal de São Paulo, São Paulo.

COX, R. M. Administration and application of the APHAB. Hear J., v.50, n.4, p.32-48, 1997.

COX, R. M.; ALEXANDER, G. C. The abbreviated profile of hearing aid benefit. Ear Hear, v.6, n.2, p.176$183,1995$.

GORDO, A. Estudo comparativo do desempenho de próteses auditivas com circuito K-AMP e processamento automático do sinal. 1998. Dissertação (Mestrado) - Universidade Federal de São Paulo, São Paulo.
NEWMAN, C. W. Digital signal processing hearing aids. Arch. Otolaryngol Head Neck Surg., v.126, n.11, p.1397-1399, 2000.

VALENTE, M.; VLIET, D. V.The Independent Hearing Aid Fitting Forum (IHAFF) Protocol. Trends in Amplification, v.2, n.1, p.6-35, 1997.

VALENTE, M. et al. Differences in performance between Oticon MultiFocus and Resound BT2-E Hearing Aids. J. Am. Acad. Audiol., v.126, n.8, p.280-293, 1997.

WALDEN, B. E. et al. Comparison of benefit provided by different hearing aid tecnologies. J. Am. Acad. Audiol., v.11, p.540-560, 2000.

WEINSTEIN, B. E. Outcome measure in the hearing aid fitting/selection process. Trends in Amplification, v.2, n.4, p.117-137, 1997.

YUED, B. Digital hearing aids. Arch. Otolaryngol Head Neck Surg., v.126, n.11, p.1394-1397, 2000. 


\section{ABREVIATE PROFILE OF HEARING AID BENEFIT - A.P.H.A.B}

Instruçóes: Por favor, circule as respostas que mais se aproximam de seu dia-a-dia. Note que cada escolha inclui numa porcentagem. Você pode usar isto para decidir sua resposta. Por exemplo, se um item for verdadeiro por volta de $75 \%$ das vezes circule a letra C. Se não tiver experienciado a situação descrita, tente pensar em uma situação similar a esta. Se você não tiver idéia, deixa em branco.

A sempre $99 \%$

B quase sempre $87 \%$

C geralmente $75 \%$

D metade das vezes $50 \%$

E às vezes $25 \%$

F raramente $12 \%$

G nunca $1 \%$

Sem a prótese

Com a prótese

\begin{tabular}{|c|c|c|}
\hline $\begin{array}{l}\text { 1. Quando estou no supermercado, conversando com o caixa, } \\
\text { eu posso seguir a conversa... }\end{array}$ & A B C D EF G & A B C D E F G \\
\hline $\begin{array}{l}\text { 2. Eu perco informação quando estou em uma aula, curso } \\
\text { ou palestra... }\end{array}$ & A B C D E F G & A B C D E F G \\
\hline $\begin{array}{l}\text { 3. Sons inesperados, como um alarme de um carro, são } \\
\text { descnfortáveis... }\end{array}$ & A B C D E F G & A B C D E F G \\
\hline $\begin{array}{l}\text { 4. Eu tenho dificuldade em ouvir a conversa com um dos } \\
\text { meus familiares em casa... }\end{array}$ & A B C D E F G & A B C D E F G \\
\hline $\begin{array}{l}\text { 5. Tenho dificuldade para entender um diálogo no cinema } \\
\text { ou no teatro... }\end{array}$ & A B C D E F G & A B C D E F G \\
\hline $\begin{array}{l}\text { 6. Quando estou ouvindo as notícias no rádio do carro e os membros } \\
\text { da família estão falando, tenho dificuldade para entender as } \\
\text { notícias... }\end{array}$ & A B C D E F G & A B C D E F G \\
\hline $\begin{array}{l}\text { 7. Quando estou numa mesa de jantar com várias pessoas e estou } \\
\text { tentando conversar com uma delas, é difícil compreender a fala... }\end{array}$ & A B C D E F G & A B C D E F G \\
\hline 8. Os sons do trânsito são muito intenssos... & A B C D E F G & A B C D E F G \\
\hline $\begin{array}{l}\text { 9. Quando estou conversando com alguém em uma sala ampla } \\
\text { vazia, eu compreendo as palavras... }\end{array}$ & A B C D E F G & A B C D E F G \\
\hline $\begin{array}{l}\text { 10. Quando estou em uma sala pequena, perguntando ou } \\
\text { respondendo questóes, tenho dificuldade de seguir a conversa... }\end{array}$ & A B C D E F G & A B C D E F G \\
\hline $\begin{array}{l}\text { 11. Quando estou em um teatro assistindo a um filme ou peça e } \\
\text { as pessoas ao meu redor estão sussurrando ou amassando papéis } \\
\text { de bala, eu ainda posso entender o diálogo... }\end{array}$ & A B C D E F G & A B C D E F G \\
\hline $\begin{array}{l}\text { 12.Quando estou conversando em fraca intensidade com um } \\
\text { amigo, tenho dificuldade de compreensão... }\end{array}$ & A B C D E F G & A B C D E F G \\
\hline $\begin{array}{l}\text { 13. Os sons da água corrente, como na pia da cozinha, no } \\
\text { banheiro ou no chuveiro são desconfortavelmente intensos... }\end{array}$ & A B C D E F G & A B C D E F G \\
\hline $\begin{array}{l}\text { 14. Quando um falante se dirige a um pequeno grupo e todos } \\
\text { estão ouvindo silenciosamente, tenho que me esforçar para } \\
\text { compreender... }\end{array}$ & A B C D E F G & A B C D E F G \\
\hline $\begin{array}{l}\text { 15. Quando estou conversando com meu médico na sala de } \\
\text { exame, é difícil acompanhar a conversa... }\end{array}$ & A B C D E F G & A B C D E F G \\
\hline $\begin{array}{l}\text { 16. Eu posso entender a conversa mesmo quando várias pessoas } \\
\text { estấo falando ao mesmo tempo... }\end{array}$ & A B C D E F G & A B C D E F G \\
\hline 17. Os barulhos de uma construção são desconfortavelmente intensos... & A B C D E F G & A B C D E F G \\
\hline 18. É difícil para eu entender o que é dito em palestra ou em igrejas... & A B C D E F G & A B C D E F G \\
\hline 19. Eu posso me comunicar com os outros no meio da multidão... & A B C D E F G & A B C D E F G \\
\hline $\begin{array}{l}\text { 20. O som de uma sirene próxima é tão intenso que preciso cobrir } \\
\text { minha orelhas... }\end{array}$ & A B C D E F G & A B C D E F G \\
\hline $\begin{array}{l}\text { 21. Eu posso seguir as palavras de um sermão em uma missa ou } \\
\text { culto religioso... }\end{array}$ & A B C D E F G & A B C D E F G \\
\hline 22. O som de uma brecada de carro é desconfortavelmente intenso... & A B C D E F G & A B C D E F G \\
\hline $\begin{array}{l}\text { 23. Conversando com outra pessoa em ambiente silencioso, eu preciso } \\
\text { pedir para ela repetir o que foi dito... }\end{array}$ & & \\
\hline $\begin{array}{l}\text { 24. Tenho dificuldade para compreender o que os outros dizem quando } \\
\text { o ar condicionado ou o ventilador está ligado... }\end{array}$ & A B C D E F G & A B C D E F G \\
\hline
\end{tabular}

